\title{
Revisión de métodos para la clasificación de fallas superficiales en pavimentos flexibles ${ }^{*}$
}

\author{
Norma Ximena Ríos Cotazo - Bladimir Bacca Cortés ${ }^{b}$ \\ - Eduardo Caicedo Bravoc - Armando Orobio Quiñónez ${ }^{\mathrm{d}}$
}

\begin{abstract}
Resumen: El estado de la infraestructura vial impacta el entorno social, económico y político de una nación. La evaluación de la condición superficial del pavimento es esencial para planificar intervenciones oportunas y eficaces. Las acciones oportunas evitan sobrecostos de operación, impiden el deterioro no controlado y disminuyen los inconvenientes operacionales y de seguridad. El problema expuesto plantea la inquietud de estudiar alternativas para evaluar el estado del pavimento, por lo cual un gran número de investigaciones sobre detección automática de fallas superficiales en pavimentos flexibles a través de técnicas de procesamiento de imágenes han sido desarrolladas. El objetivo de este artículo es revisary analizar estos aportes. Sobre la base de la revisión, se concluyó que el rendimiento de este tipo de sistemas está determinado por dos factores: la recopilación de los datos y su procesamiento. El análisis presentado se despliega en función de estos factores. Se considera oportuno el desarrollo de sistemas que aprovechen las cualidades de diferentes sensores en la adquisición de datos y que integren la detección y clasificación de variedad de fallas incluyendo datos de severidad.
\end{abstract}

Palabras clave: pavimentos flexibles; fallas superficiales; multisensorial; visión artificial

Recibido: 02/12/2019 Aceptado: 25/06/2020

Disponible en línea: 09/12/2020

Cómo citar: N. X. Ríos Cotazo, B. Bacca Cortés, E. Caicedo Bravo, y A. Orobio Quiñónez, «Revisión de métodos para la clasificación de fallas superficiales en pavimentos flexibles», Cien.Ing.Neogranadina, vol. 30, n. 2 , jul. 2020.

* Artículo de revisión.

a Mg. Universidad del Valle. Estudiante de Doctorado, Universidad del Valle. Cali, Colombia. Correo electrónico: ximena.rios@correounivalle.edu.co ORCID: https://orcid.org/0000-0002-4965-887X

b Ph. D. Universitat de Girona. Profesor Titular Universidad del Valle. Cali, Colombia. Correo electrónico: bladimir.bacca@correounivalle.edu.co ORCID: https://orcid.org/0000-0003-0113-4134

c Ph. D. Universidad Politécnica de Madrid. Profesor Titular Universidad del Valle. Cali, Colombia. Correo electrónico: eduardo.caicedo@correounivalle.edu.co. ORCID: https://orcid.org/0000-0003-07272917

d Ph. D. West Virginia University. Profesor Titular Universidad del Valle Cali, Colombia. Correo electrónico: armando.orobio@correounivalle.edu.co. ORCID: https://orcid.org/0000-0001-7166-3061 


\section{Review of methods for classifying surface faults in flexible pavements}

Abstract: The status of the road infrastructure affects the social, economic, and political environment of a nation. Evaluation of the pavement surface condition is essential to plan timely and effective interventions. Timely actions avoid operating cost overruns, prevent uncontrolled deterioration and reduce operational and safety inconveniences. The problem raises the concern of studying alternatives to evaluate the status of pavement, for which a large number of investigations on automatic detection of surface flaws in flexible pavements through image processing techniques have been developed. . The objective of this article is to review and analyze these contributions. Based on the review, it was concluded that the performance of this type of systems is determined by two factors: data collection and processing. The analysis presented herein unfolds based on these factors. The development of systems that take advantage of the qualities of different sensors in data acquisition and that integrate the detection and classification of a variety of faults including severity data is considered opportune.

Keywords: Flexible pavements; surface faults; multisensory; artificial vision 


\section{Introducción}

La infraestructura vial constituye un factor de gran influencia en el progreso de una región [1], el mal estado de las vías disminuye la calidad del servicio, aumenta los costos de transporte y afecta la seguridad vial. El punto de inicio para determinar la necesidad de intervención en los pavimentos es la evaluación de su condición [2], [3]. Esta evaluación se puede realizar de forma manual, para lo cual se requiere personal técnico calificado, el proceso es lento y propenso a subjetividad. También es posible utilizar equipos automáticos para recopilar datos del estado del pavimento y luego aplicar técnicas de procesamiento de datos para detectar las fallas y clasificarlas; estos sistemas, además de ser confiables, no destructivos y seguros para la manipulación humana, hacen posible la inspección de tramos viales amplios y reducen el tiempo requerido para la evaluación.

A pesar de que se encuentran muchas investigaciones en reconocimiento y clasificación automática de fallas en pavimentos, con resultados significativos, aun existe la posibilidad de encontrar opciones que puedan mejorar el rendimiento de estos sistemas. Este artículo pretende determinar la tendencia en este tipo de sistemas. En esta revisión se han considerado dos etapas fundamentales: la adquisición de datos y el procesamiento de los mismos. La adquisición de datos hace referencia a los instrumentos que se utilizan para la recopilación de la información tomada sobre la superficie del pavimento, y el procesamiento de datos comprende las técnicas utilizadas para operar los datos adquiridos y determinar la condición de la superficie.

El artículo está organizado de la siguiente manera. En la sección 1 se presentan los sistemas utilizados para adquirir los datos de la superficie del pavimento, examinados en cuatro grupos: sistemas láser, cámaras digitales, sistemas comerciales y otros métodos. En la sección 2 se exponen las técnicas de procesamiento de datos utilizadas para la detección de fallas, revisadas en dos grupos: detección de fallas basada en técnicas de visión por computador y detección de fallas basada en técnicas de aprendizaje automático. En la sección 3, se proporcionan discusiones detalladas y finalmente las conclusiones.

\section{Sistemas de adquisición de datos}

Para la evaluación vial se utilizan diversos equipos en la recopilación de los datos. La Tabla 1 registra los dispositivos utilizados en las investigaciones consultadas. Separamos estos dispositivos en cuatro grupos, como se muestra en la Figura 1: sistemas láser, cámaras digitales, sistemas comerciales y otros métodos.

SISTEMAS DE ADQUISICIÓN DE DATOS



Figura 1. Sistemas de adquisición de datos para detección de fallas superficiales. Fuente: elaboración propia. 
Tabla 1. Sistemas de adquisición de datos para detectar fallas superficiales

\section{Sistema de adquisición}

Autor(es)

Sistemas
láser $\quad \frac{\text { Triangulación }}{\text { Tiempo de vuelo }} \frac{[5][6][7][8]}{\text { Luz estructurada }} \frac{[10][11][12][13]}{[90}$

\begin{tabular}{|c|c|c|}
\hline \multirow{4}{*}{$\begin{array}{l}\text { Cámaras } \\
\text { digitales }\end{array}$} & $\begin{array}{l}\text { Cámaras de } \\
\text { escaneo Lineal }\end{array}$ & [15] [16] [17] [18] [19] \\
\hline & $\begin{array}{l}\text { Cámaras de } \\
\text { escaneo por área }\end{array}$ & $\begin{array}{l}{[20][21][22][23][24]} \\
{[25][26][27][28][29]} \\
{[30][31][32][33][34]} \\
{[35][36]}\end{array}$ \\
\hline & Multiespectrales & [37] [38] [39] [40] \\
\hline & Video & [41] [42] [43] [44] \\
\hline \multirow{4}{*}{ Otros } & $\begin{array}{l}\text { Sensor RGB-D } \\
\text { (Red Green Blue - } \\
\text { Deep) }\end{array}$ & [48] \\
\hline & $\begin{array}{l}\text { Sensor } \\
\text { ultrasónico }\end{array}$ & [45] \\
\hline & $\begin{array}{l}\text { Sensor de } \\
\text { profundidad }\end{array}$ & {$[46]$} \\
\hline & Acelerómetro & [47] \\
\hline
\end{tabular}

Fuente: elaboración propia.

\section{Sistemas láser}

Los sistemas láser son adecuados para la detección de fallas debido a su capacidad de obtener información de profundidad $y$, por ser poco vulnerables a las condiciones de iluminación, proporcionan una alta precisión, pero representan un recurso costoso.

Las técnicas de captación de perfiles 3D basados en los sistemas laser más habituales en evaluación de pavimentos son: tiempo de vuelo, triangulación, diferencia de fase y luz estructurada [4].

\section{Triangulación}

Investigaciones como [5] - [8] adquieren datos de la superficie de la vía mediante un escáner que usa el principio de triangulación. En este escáner el brillo del láser se examina mediante una cámara fotográfica para determinar su posición. El punto del objeto en que brilla el láser incidirá en diversos sitios del campo visual de la cámara dependiendo de la distancia [4].

\section{Tiempo de vuelo}

En [10] manejan dos escáneres láser Riegl VQ-450 basados en la tecnología de tiempo de vuelo y cuatro cámaras digitales de alta resolución para detectar tapas de alcantarilla. El escáner láser de tiempo de vuelo cronometra el tiempo que tarda un pulso de luz en recorrer la distancia al objeto y volver [4].

\section{Luz estructurada}

La técnica de luz estructurada utiliza la proyección de un patrón de luz en el objeto y analiza la deformación del patrón para determinar la posición de cada punto. La captura se realiza con una o varias cámaras fotográficas. Existen diversos métodos de luz estructurada, dentro de los cuales existen tres principales: proyección de un punto láser, proyección de una línea láser y proyección de un patrón de franjas [4]. Los valores de elevación se deducen teniendo en cuenta la deformación de la línea láser que se proyecta sobre la superficie del pavimento [10] - [13].

\section{Cámaras digitales}

Utilizar cámaras digitales en detección de fallas es muy habitual. Tienen varias ventajas, como precios razonables (dependiendo de la resolución), facilidad de uso y accesibilidad.

\section{Cámaras de escaneo lineal}

En las cámaras de escaneo lineal el sensor está compuesto por una sola fila de píxeles. Las imágenes se construyen a partir de la secuencia de filas capturadas a medida que el objeto fotografiado va pasando por el campo de visión de la cámara. Estos sistemas requieren un alto grado de precisión, por lo cual la alineación y el sincronismo son críticos. Este tipo de cámara permite obtener imágenes a alta velocidad, pero el aumento de velocidad requiere una iluminación que proporcione una imagen clara con poco tiempo de exposición [14]. 
[15] - [19] usan cámaras de escaneo lineal para adquirir los datos del pavimento. Para mejorar las condiciones de iluminación, [16] y [19] utilizan módulos de iluminación láser; en [15], se eliminan las sombras reconstruyendo la imagen a partir de imágenes complementarias capturadas con dos cámaras.

\section{Cámaras de escaneo por área}

En estas cámaras, el sensor está formado por una matriz de píxeles, de manera que una imagen bidimensional correspondiente a un área puede ser generada en un solo ciclo de exposición. La velocidad de lectura de las cámaras de escaneo por área es menor que la de las cámaras lineales [14], lo que reduce la tasa de captación de datos. También requieren una extensión en el montaje para que su campo de visión no sea obstruido y proporcionar una iluminación uniforme del área de escaneo. Numerosas investigaciones han utilizado imágenes de pavimentos capturadas con este tipo de cámara. Para este trabajo se agruparon como: bases de datos, modelos 3D y vant (vehículo aéreo no tripulado), que se describen a continuación.

Bases de datos: algunos investigadores no entregan detalles sobre los dispositivos de adquisición, utilizan imágenes $2 \mathrm{D}$ almacenadas en bases de datos y se enfocan en elaborados algoritmos de procesamiento [20] - [30].

Modelos 3D: en la etapa de adquisición también se incorporan los sistemas que obtienen modelos en 3D partiendo de imágenes en dos dimensiones. [31] utiliza una técnica fotogramétrica llamada SFM (structure from motion). [32] utiliza cámaras de visión estéreo para obtener un mapeo espacial. Los sistemas de visión estéreo presentan limitaciones asociadas con el emparejamiento de píxeles y las áreas de objetos parcialmente obstruidos, lo cual genera un efecto negativo en la precisión del mapeo.

Vehículos aéreos no tripulados: el uso de imágenes capturadas desde VANT también ha despertado interés en varios investigadores [33] - [36]. Las ventajas de los sistemas VANT son su bajo costo, velocidad, maniobrabilidad y seguridad en la recolección de imágenes; sin embargo, se debe tener precaución debido a que a cierta altura de vuelo la resolución espacial de las imágenes puede limitar la capacidad de detectar fallas individuales. No obstante, las imágenes aéreas se pueden usar para evaluar el estado general de las superficies de pavimento de una manera rápida, rentable y segura.

\section{Imágenes multiespectrales}

A diferencia de las imágenes digitales habituales que generalmente tienen uno o tres canales que proporcionan características espectrales limitadas, las imágenes multiespectrales tienen una amplia cobertura de longitud de onda que proporciona características espectrales detalladas. Con la incorporación de sensores capaces de capturar este tipo de imágenes, se ha propiciado el desarrollo en investigaciones que utilizan la relación entre los datos espectrales y el estado de la superficie del pavimento. [37] utiliza imágenes multiespectrales para detectar fisuras y baches; las imágenes son capturadas con una matriz de múltiples cámaras micro-MCA (por su sigla en inglés, Multispectral Camera Array). Diseñada por Tetracam, esta matriz de cámaras captura 12 bandas que abarcan longitudes de onda entre $450 \mathrm{~nm}-1000 \mathrm{~nm}$. [38] utiliza un conjunto de imágenes multiespectrales obtenidas del centro de análisis de datos de la tierra (EDAC, por su sigla en inglés Earth Data Analysis Center); estas fotografías aéreas fueron recopiladas con el sistema Zeiss/Intergraph DMC (del inglés, Digital Mapping Camera) y están compuestas por 3 bandas espectrales que abarcan longitudes de onda entre 0.4 um-0.7 um. [39] utiliza imágenes multiespectrales obtenidas con el sensor Mivis (del inglés, Multispectral Infrared and Visible Imaging Spectrometer) utilizando 10 bandas que abarcan longitudes de onda entre 8.2 um-12.7 um. [40] utiliza imágenes hiperespectrales adquiridas con el sensor Compact Airborne Spectrographic Imager, CASI-1500 estas imágenes contienen hasta 24 bandas espectrales que abarcan longitudes de onda entre $380.1 \mathrm{~nm}-1033.1 \mathrm{~nm}$.

Las imágenes multiespectrales proporcionan información sobre las propiedades de la superficie que permiten estimar la condición de la vía; sin embargo, se requiere más investigación para el desarrollo de herramientas que ayuden a sobreponerse 
a dificultades relacionadas con las variaciones en la reflectividad, causadas por las sombras de la rugosidad de la superficie y la alta circulación en las vías.

\section{Cámara de video}

Otros investigadores utilizan datos tomados desde video para detectar fallas [41] - [44]. Una desventaja en el uso de video es que la resolución de las imágenes está asociada a la velocidad del vehículo desde el cual se está tomando la señal. La adquisición debe realizarse usando una buena sincronización para garantizar la confiabilidad del sistema. La principal ventaja es la sencillez en la adquisición.

\section{Otros métodos}

Otros autores proponen sistemas variados para indicar fallas en la vía. [45] discute un método para identificar baches y ondulaciones que usa sensores ultrasónicos. [46] propone un sistema que detecta pequeñas irregularidades en la vía usando sensores de profundidad. En [47], el sistema se basa en la recolección de datos de un acelerómetro y de un GPS. [48] utiliza un sistema que usa un sensor RGB-D, Microsoft Kinect. La principal limitación de estos sistemas es el rango del ancho del sensor y la profundidad de las fallas que puede detectar.

\section{Sistemas comerciales}

Compañías internacionales han equipado vehículos para capturar datos de la superficie del pavimento con el fin de ofrecer servicios que van desde la recopilación de datos de campo hasta la interpretación y análisis de los mismos. ARAN (Automatic Road Analyzer), de Fugro [49], es un vehículo equipado con un sistema de recolección de datos y software de procesamiento; su diseño es modular y se puede configurar a la necesidad del cliente. DHDv (Digital Highway Data Vehicle), de WayLink [50], es un vehículo que integra varios subsistemas de captura de datos, posicionamiento y análisis de fallas en las vías. Leica Geosystems ofrece una plataforma de captura llamada Pegasus [51]; este sistema captura datos de nubes de puntos e imágenes y algunos datos de sensores adicionales. Su aplicación principal es de mapeo en infraestructura, aunque el fabricante deja abierta la posibilidad de su uso en detección de fallas. REAL (del inglés, Road Excellent Automatic Logging), de Pasco [52], es un vehículo que cuenta con un sistema de registro automático de vías. El MFv ( Multi-Functional Vehicle), de Dynatest [53], también cuenta con varios módulos de adquisición de datos acoplados. Fiona [54] es un vehículo para toma de datos en carreteras desarrollado por RaurosGroup, que permite grabación de imágenes y toma de datos geométricos. La principal ventaja del uso de estos equipos es la recolección rápida y confiable de la información. Pero la fabricación y el soporte son costosos. Además, los datos obtenidos son de gran tamaño y la decisión automática del tipo de falla es una tarea cuantiosa que requiere de herramientas potentes y software especializado.

\section{Procesamientos de datos}

Las investigaciones iniciales en detección de fallas visibles mediante análisis automático se enfocaron en técnicas de visión por computador fundamentadas en distintas disciplinas como la geometría, la estadística, la física, entre otras. Los enfoques modernos combinan técnicas de procesamiento de imágenes y aprendizaje automático dando resultados satisfactorios bajo condiciones delimitadas. La Tabla 2 registra el tipo de procesamiento realizado en los trabajos consultados. Separamos estas técnicas en dos grupos, como se muestra en la Figura 2: detección de fallas basada en técnicas de visión por computador y detección de fallas basada en técnicas de aprendizaje automático. 


\section{TÉCNICAS DE PROCESAMIENTO PARA DETECCIÓN DE FALLAS SUPERFICIALES}

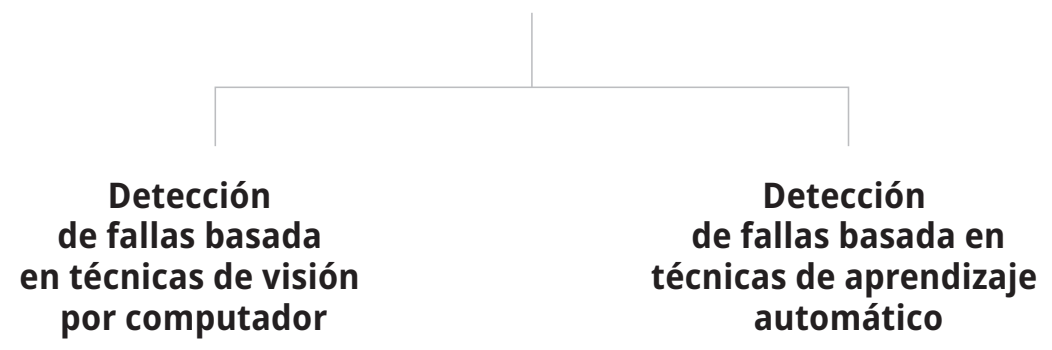

Técnicas de
descomposición

Enfoque basado

en semillas

Métodos de umbral

Otros métodos

\begin{abstract}
Detección
de fallas basada en

automático
\end{abstract}

Máquinas de

soporte vectorial

Árboles de decisión

Redes neuronales

Aprendizaje profundo

Figura 2. Técnicas de procesamiento para detección de fallas superficiales.

Fuente: elaboración propia. 
Tabla 2. Técnicas de procesamiento utilizadas en sistemas para detectar fallas superficiales en pavimentos flexibles, según los trabajos consultados

\begin{tabular}{|c|c|c|}
\hline \multicolumn{2}{|c|}{ Técnicas de procesamiento } & Autor(es) \\
\hline \multirow{4}{*}{$\begin{array}{l}\text { Detección de } \\
\text { fallas basada en } \\
\text { técnicas de visión } \\
\text { por computador }\end{array}$} & $\begin{array}{l}\text { Técnicas de } \\
\text { descomposición }\end{array}$ & $\begin{array}{l}{[10][14][56][57]} \\
{[58][59]}\end{array}$ \\
\hline & $\begin{array}{l}\text { Enfoque basado } \\
\text { en semillas }\end{array}$ & {$[17][18][19]$} \\
\hline & $\begin{array}{l}\text { Métodos de } \\
\text { umbral }\end{array}$ & {$[11][39][42][60]$} \\
\hline & Otros métodos & $\begin{array}{l}{[6][7][33][34]} \\
{[38][40][44]}\end{array}$ \\
\hline \multirow{4}{*}{$\begin{array}{l}\text { Detección de } \\
\text { fallas basada } \\
\text { en técnicas de } \\
\text { aprendizaje } \\
\text { automático }\end{array}$} & $\begin{array}{l}\text { Máquinas de } \\
\text { soporte vectorial }\end{array}$ & $\begin{array}{l}{[21][23][25][26]} \\
{[35][37][43][62]}\end{array}$ \\
\hline & $\begin{array}{l}\text { Árboles de } \\
\text { decisión }\end{array}$ & $\begin{array}{l}{[9][22][24][25]} \\
{[30][37]}\end{array}$ \\
\hline & Redes neuronales & $\begin{array}{l}{[8][25][26][29]} \\
{[36][37]}\end{array}$ \\
\hline & $\begin{array}{l}\text { Aprendizaje } \\
\text { profundo }\end{array}$ & $\begin{array}{l}{[20][27][28][63]} \\
{[64]}\end{array}$ \\
\hline
\end{tabular}

Fuente: elaboración propia.

\section{Detección de fallas basado en técnicas de visión por computador}

La visión por computador es una rama de la inteligencia computacional que busca la deducción automática de la estructura del mundo real a partir de la comprensión de la información de una o varias imágenes [55]. Las investigaciones consultadas se han separado en cuatro grupos: análisis mediante descomposición, algoritmos de crecimientos de semillas, aplicación de umbral y otros métodos. Como otros métodos se incluyen los algoritmos de detección de bordes, el análisis usando la emisividad del pavimento, el análisis de contornos, entre otros.

\section{Técnicas de descomposición}

El análisis mediante la transformación de la señal en componentes más representativos siempre ha estado presente en la ingeniería. La idea ha evolucionado con el tiempo y las investigaciones más recientes nos llevan a una variedad de transformaciones como la transformada Wavelet o la transformada Hough, entre otras, que constituyen una herramienta matemática que simplifica los cálculos y puede usarse para abordar el problema de detección de fallas en pavimentos, como se observa en [10], [12], [56] - [59].

\section{Enfoque basado en semillas}

Algunos investigadores han abordado el enfoque basado en semillas [17] - [19]. Estos métodos comparten dos pasos comunes: se extraen los puntos que probablemente pertenezcan a una falla (puntos semilla) y se unen los caminos entre semillas mediante técnicas de crecimiento. La simplicidad del enfoque basado en semillas es su principal ventaja; pero los resultados de la detección dependen de la elección de las semillas, siendo esto una desventaja, ya que el ruido en la imagen puede hacer que las semillas queden mal inicializadas.

\section{Métodos de umbral}

Los métodos de umbral consisten en fijar unos límites para separar los rasgos de interés; estos métodos generalmente son sensibles al ruido, aunque se han propuesto alternativas que intentan mejorar su desempeño, en [11], [39], [42] y [60]. El problema en estos métodos es encontrar el valor del umbral que separe los rasgos de interés. En términos generales su simplicidad es una ventaja, pero pueden ser sensibles al ruido y son altamente dependientes de la iluminación. Estos métodos son adecuados cuando hay una clara diferencia entre los rasgos que se desean separar.

\section{Otros métodos}

Varias alternativas de procesamiento se han planteado en esta sección. El uso de la respuesta espectral [38] [40] como herramienta de análisis tiene la desventaja de que aun no se han obtenido resultados satisfactorios en la detección de fallas 
específicas; sin embargo, es útil para obtener índices globales de la condición de la vía. Otras alternativas, como el uso de operadores para detección de bordes [33] - [34] y [44], presentan desventajas al ser sensibles al ruido y a determinadas orientaciones dependiendo del operador, pero su ventaja es la simplicidad. Los contornos activos [7] pueden realizar una buena separación de las fallas, tienen la ventaja de ser robustos frente al ruido y a la existencia de bordes falsos, pero su desventaja es la necesidad de inicializar con una cercanía al objeto y englobándolo, además de presentar conflictos con las concavidades. El uso de un sistema Fuzzy [6] tiene la ventaja de permitir relacionar entradas y salidas sin conocer el modelo matemático que rige su funcionamiento; por lo mismo, el análisis de cualquier propiedad del sistema es complejo.

\section{Aprendizaje automático}

El aprendizaje automático (machine learning) es otra rama de la inteligencia computacional. Consiste en crear programas capaces de generalizar la relación entre entradas y salidas para automáticamente mejorar sus algoritmos [61]. En los últimos años, con el desarrollo del aprendizaje automático, se introdujeron en el campo de detección automática de fallas en el pavimento algoritmos como máquinas de soporte vectorial (svm, del inglés, Support Vector Machines), árboles de decisión, redes neuronales y aprendizaje profundo, constituyendo una alternativa confiable.

\section{Máquinas de soporte vectorial}

Las svm son un método de clasificación donde la idea principal es construir hiperplanos como superficies de decisión, de tal manera que el margen de separación entre los ejemplos positivos y negativos se maximice [65]. Algunas publicaciones, como [19], [21], [23], [25], [43], [35] y [62], muestran que las Svm arrojan resultados satisfactorios en la detección de fallas en pavimentos.

Dentro de las fortalezas de las svm tenemos que su modelamiento no necesita la totalidad de puntos disponibles del conjunto de entrenamiento para hallar la separación entre clases, lo cual representa una ventaja frente a otros métodos que utilizan un porcentaje alto de las muestras del conjunto de entrenamiento. Mientras la mayoría de los métodos de aprendizaje se centran en minimizar los errores generados a partir de los ejemplos de entrenamiento, las SVM se centran en la minimización del denominado riesgo estructural, el cual ha mostrado un mejor desempeño, ya que minimiza un límite superior en lugar de minimizar el error sobre los datos de entrenamiento. Así mismo, la capacidad de generalización y el proceso de entrenamiento de la máquina no dependen necesariamente del número de atributos, lo que permite un buen comportamiento en problemas de alta dimensionalidad. Otros aspectos favorables son: tienden a ser menos propensas a problemas de sobrentrenamiento, la complejidad está caracterizada por el número de vectores de soporte en lugar de la dimensionalidad del espacio transformado, el error es independiente de la dimensionalidad, la solución es global y no hay óptimo local como en las redes neuronales. En sentido opuesto, como debilidades se puede mencionar que se necesita una buena función kernel, es decir, se necesitan metodologías eficientes para estimar los parámetros de la svm. De igual forma, aunque las svm usan funciones de decisión directa, el problema de las multiclase no es sencillo porque se tienen muchas formulaciones.

\section{Árboles de decisión}

Los árboles de decisión son una técnica de clasificación que utiliza aprendizaje inductivo. Se trata de un método para aproximar funciones de valores discretos, capaz de expresar hipótesis disyuntivas a partir de un conjunto de datos de entrenamiento [66]. Los resultados publicados en [9], [19], [24], [30] y [37] muestran que los árboles de decisión pueden ser útiles en la detección de fallas.

Los árboles de decisión tienen la ventaja de realizar de forma implícita un proceso de selección de las variables más significativas. Una vez construido el árbol, aquellos atributos que no intervienen en ninguna condición pueden descartarse, reduciéndose el tamaño del espacio de características. Otra ventaja es que pueden tratar con atributos continuos o discretos, permiten tratar relaciones no lineales entre características y clases, las muestras de aprendizaje de una misma clase no tienen que 
ser homogéneas entre sí, son tolerantes al ruido y a atributos no significativos. Entre las desventajas se puede mencionar que no son tan precisos como otros métodos, no detectan correlaciones, tratan de dividir el dominio de los atributos en regiones rectangulares, pueden tener problemas de sobreaprendizaje, además son dependientes de las muestras que se les suministran para el aprendizaje.

\section{Redes neuronales}

Una red neuronal (RN) es un sistema de procesamiento de información basado en un conjunto estructurado de elementos de procesamiento interconectados. La habilidad de procesamiento de la red está relacionada con los pesos de las conexiones entre los elementos; estos se obtienen a través de un proceso de adaptación a un conjunto de muestras de entrenamiento [67]. Investigaciones como [8], [26], [29] y [36] revelan la potencialidad de las redes neuronales en la solución del problema de detección de fallas en pavimentos.

A favor de las redes neuronales cabe mencionar que son robustas, ya que pueden responder de manera aceptable, aun si fallan algunos elementos de procesamiento. También son flexibles, puesto que pueden manejar variaciones no importantes en la información de entrada como señales con ruido, distorsionadas o incompletas. Otro punto a favor es que para utilizar RN no es necesario conocer los detalles matemáticos, solo se requiere estar familiarizado con los datos de trabajo. Dentro de los aspectos desfavorables generales tenemos que el tiempo de entrenamiento puede ser elevado, el aprendizaje para grandes tareas puede resultar complejo, demandan la definición de varios parámetros antes de aplicar la metodología, pueden tener problemas de sobreaprendizaje y para adicionar nuevo conocimiento es necesario cambiar las interacciones entre muchas unidades de procesamiento.

\section{Aprendizaje profundo}

Mientras que los métodos de inteligencia computacional tradicionales requieren que las características de la imagen estén especificadas manualmente, el aprendizaje profundo proporciona una solución nueva y prometedora que tiene la ventaja de realizar la extracción de características y los procesos de clasificación de manera integrada y totalmente automatizada. Esto ha despertado el interés de los investigadores en emplear estas arquitecturas en la detección de fallas en el pavimento. Publicaciones como [20], [27] - [28] y [63] - [64] muestran resultados con buenos índices de desempeño.

El principal problema del uso de una arquitectura profunda es conseguir una buena representación de la entrada; además, si una solución se representa con una arquitectura muy grande podrían necesitarse muchos ejemplos para el entrenamiento, pero una profundidad insuficiente puede ser perjudicial para el aprendizaje. Se consideran ventajas su posibilidad de ser aplicado a funciones con representaciones abstractas de información y su prometedora capacidad de aprendizaje automático.

\section{Discusión}

En la etapa de adquisición de datos se han probado varios dispositivos, que han demostrado su utilidad en la evaluación de pavimentos. Todos ofrecen confiabilidad y eficiencia bajo ciertas condiciones. Los sistemas comerciales, [50] - [54], usan una combinación de cámaras digitales apoyada por varios sensores, lo que los hace más robustos y al mismo tiempo costosos. Las imágenes digitales 2D se utilizan con frecuencia, como se observa en [15] - [30] y [34] - [36]; sin embargo, los métodos 2D no pueden detectar algunos tipos de falla debido a la falta de información de profundidad y pueden tener dificultad para discriminar áreas oscuras, como sombras e iluminación deficiente. Para hacer frente a estas desventajas, se propusieron sistemas que obtienen modelos 3D partiendo de imágenes 2D, [31] - [33], y también se incorporan los sistemas de escaneo láser que pueden recopilar perfiles 3D para construir superficies de pavimento [5] [6], [9] - [13]. Como una alternativa a los métodos de evaluación con sensores montados en vehículos terrestres, algunos investigadores han propuesto la captura de datos utilizando vant [31], [33] - [36]. Los VANT presentan ventajas como su bajo costo, velocidad, seguridad, además no interfieren con el tránsito y tienen la posibilidad de vuelo en modo autónomo o dirigido; presentan inconvenientes 
debido a las obstrucciones por objetos presentes sobre la superficie en el momento de la recolección de datos; también tienen una limitación en la resolución, debido a la distancia de muestreo en tierra, lo que en términos generales limita la capacidad de detección. Los sensores multiespectrales también ofrecen una alternativa en la etapa de adquisición de datos. Es posible determinar la tasa global de fallas en las vías a través de la respuesta espectral [37] - [40], pero se requiere más investigación para el desarrollo de herramientas que permitan la detección y cuantificación de las fallas individuales; de las investigaciones consultadas, solo [37] evalúa fallas individuales a partir de imágenes multiespectrales; estos desarrollos podrían usarse como complemento a la inspección de campo o para la evaluación general de la gestión de las agencias que administran las vías.

En la etapa de procesamiento de datos, se han probado varias técnicas. Cada técnica ofrece ventajas y también presenta desventajas. El enfoque basado en semillas es simple, pero los resultados dependen de una buena elección de las semillas y esta elección puede ser afectada por el ruido; los métodos del umbral también son simples, pero sensibles al ruido y altamente dependientes de picos; los operadores para detección de bordes son simples, pero sensibles al ruido y a determinadas orientaciones dependiendo del operador; los contornos activos son robustos frente al ruido y a la existencia de bordes falsos, pero requieren ser inicializados con una cercanía al objeto y englobándolo, además de presentar conflictos con las concavidades; los sistemas Fuzzy permiten relacionar entradas y salidas sin conocer el modelo matemático que rige su funcionamiento, pero el análisis de cualquier propiedad del sistema es complejo; las svm manejan bien el problema de la alta dimensionalidad, pero necesitan una buena función kernel; los árboles son tolerantes al ruido y a atributos no significativos, pero no detectan correlaciones; las RN son robustas y flexibles, pero el tiempo de entrenamiento puede ser elevado, y el aprendizaje profundo tiene la posibilidad de ser empleado en aplicaciones donde se requieren representaciones abstractas de información, pero es laborioso obtener una buena representación de la entrada.
En la Tabla 3, la primera columna corresponde a un intervalo de tiempo en años, la segunda y la tercera columna relacionan el porcentaje de uso de técnicas de visión por computador con el aprendizaje automático en estos intervalos. Se observa que en los primeros años el problema de detección de fallas se abordó utilizando técnicas de visión por computador, pero en los últimos años las técnicas de aprendizaje automático se convirtieron en las más utilizadas. Esto se debe a tres factores que impulsaron el aprendizaje automático: los avances en hardware con el desarrollo de GPU rápidas y masivamente paralelas, la disponibilidad de grandes conjuntos de datos que no podrían haberse recolectado sin internet y de los que no se disponía con anterioridad, y el planteamiento de algoritmos avanzados. Cuando estas mejoras comenzaron a permitir modelos de entrenamiento más profundos, el interés de los investigadores se volcó hacia el aprendizaje automático. Cabe resaltar que las técnicas de visión por computador aun son utilizadas en este tipo de sistemas, pero no como eje central, sino integradas como etapas de preprocesamiento.

Tabla 3. Procesamiento de datos en sistemas para detectar fallas superficiales por año

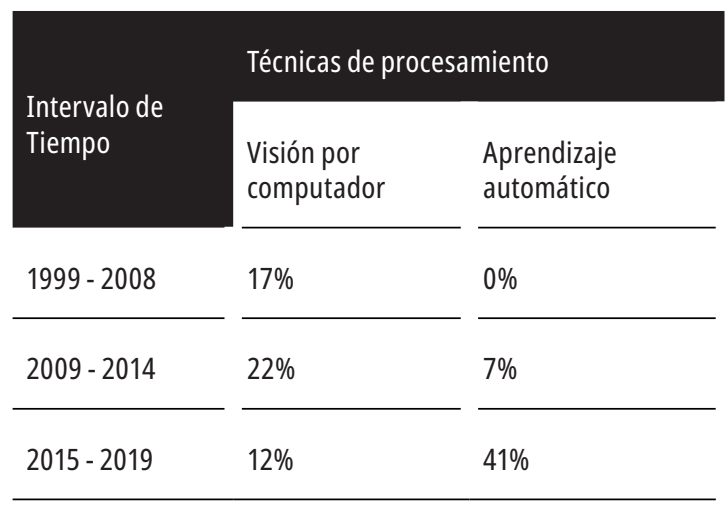

Fuente: elaboración propia.

La Tabla 4 registra la información de rendimiento de los sistemas revisados. La primera columna indica la técnica de procesamiento usada en la investigación, la segunda corresponde a la referencia citada, la tercera nos indica el número de imágenes usadas para el análisis y la cuarta corresponde al criterio de evaluación. Una comparación general en términos de desempeño sería 
improcedente, dado que no todos los autores utilizan los mismos criterios de evaluación; además, la cantidad de imágenes de prueba es diferente y existen variaciones en el tipo de falla o fallas detectadas. De la información de la Tabla 4, podemos notar que en los desarrollos fundamentados en técnicas de visión por computador los criterios de evaluación utilizados por los autores son muy variados. Utilizan medidas como: la raíz del error cuadrático medio RMSE (por su sigla en inglés, Root Mean Square Error), la correlación, la puntuación Hausdorff, diferencias estadísticas, porcentajes de error, porcentajes de acierto, la tasa de precisión de la clasificación CAR (por su sigla en inglés, Classification Accuracy Rate) y la medida de evaluación de desempeño F1. En los desarrollos fundamentados en aprendizaje automático, además de usar un número mayor de imágenes en las pruebas, el criterio de evaluación es más uniforme, pues se utilizan dos medidas: la tasa de precisión de la clasificación CAR y el puntaje F1. La tasa de precisión de la clasificación CAR cuantifica la relación entre el número de datos correctamente clasificados y el número total de datos, la puntuación F1 cuantifica la relación entre la precisión y la detección.

Tabla 4. Medidas de desempeño

\begin{tabular}{|c|c|c|c|}
\hline Técnica de procesamiento & Autor(es) & $\begin{array}{l}\text { Número de } \\
\text { imágenes usadas } \\
\text { en el análisis }\end{array}$ & Criterio de evaluación \\
\hline \multirow{6}{*}{$\begin{array}{l}\text { Técnicas de } \\
\text { descomposición }\end{array}$} & [59] & -- & -- \\
\hline & [58] & -- & -- \\
\hline & [57] & 9 & $\mathrm{CAR}=33 \%$ \\
\hline & {$[58]$} & -- & -- \\
\hline & {$[10]$} & 48 & $\begin{array}{l}\text { Detección acertada fisuras transversales }=96.58 \% \\
\text { Detección acertada fisuras longitudinales }=93.74 \%\end{array}$ \\
\hline & {$[12]$} & -- & Puntuación Hausdorff = 94.25 \\
\hline \multirow{3}{*}{$\begin{array}{l}\text { Enfoque basado en } \\
\text { semillas }\end{array}$} & {$[18]$} & 100 & $\begin{array}{l}\text { Correlación }=0.91 \text { en FL } \\
\text { Correlación }=0.96 \text { en FT }\end{array}$ \\
\hline & {$[17]$} & 5 & $\mathrm{~F} 1$-score $=0.88$ \\
\hline & {$[19]$} & 7250 & RMSE $=0.019$ \\
\hline \multirow{4}{*}{ Métodos de umbral } & {$[60]$} & 10 & $\mathrm{CAR}=89.7 \%$ \\
\hline & [39] & -- & Detección acertada = 90\%. \\
\hline & [42] & 70 & $\mathrm{CAR}=86 \%$ \\
\hline & [11] & $\begin{array}{l}220 \\
155\end{array}$ & $\begin{array}{l}\text { CAR }=94.55 \% \text { y } 89.03 \% \\
\text { Tasa de error de reconocimiento } 5.45 \% \text { y } 10,97 \% \text {. }\end{array}$ \\
\hline \multirow{7}{*}{ Otros métodos } & {$[6]$} & -- & -- \\
\hline & [40] & 4 & Porcentaje promedio en el error de $55.5 \%$ \\
\hline & [33] & -- & -- \\
\hline & [38] & -- & Correlación = 0.95 \\
\hline & [34] & -- & Diferencia estadística de 0.05 \\
\hline & [7] & -- & -- \\
\hline & {$[44]$} & 500 & $\mathrm{CAR}=80 \%$ \\
\hline
\end{tabular}




\begin{tabular}{|c|c|c|c|}
\hline \multirow{8}{*}{$\begin{array}{l}\text { Máquinas de soporte } \\
\text { vectorial }\end{array}$} & [62] & 80 & Reconoce correctamente todas las imágenes de prueba \\
\hline & [35] & 4253 & $\mathrm{CAR}=95.51 \%$ \\
\hline & [21] & 120 & CAR $=95.7 \%$ \\
\hline & [43] & 200 & $\mathrm{CAR}=87.3 \%$ \\
\hline & [23] & 500 & $\mathrm{CAR}=93.04 \%$ \\
\hline & [25] & 1500 & $\begin{array}{l}\text { CAR }=91.91 \% \text { para svm } \\
\text { CAR }=92.62 \% \text { para una svm de mínimos cuadrados }\end{array}$ \\
\hline & [37] & 1760 & $\mathrm{CAR}=98.78 \%$ \\
\hline & [26] & 200 & $\mathrm{CAR}=88.75 \%$ \\
\hline \multirow{6}{*}{ Árboles de decisión } & [30] & 1830 & $\begin{array}{l}\text { CAR }=97.9 \% \text { para baches } \\
\text { CAR }=96.4 \% \text { para fisuras }\end{array}$ \\
\hline & [9] & -- & $\begin{array}{l}\text { CAR }=95.5 \% \\
\text { F1-score }=0.957\end{array}$ \\
\hline & [22] & 118 & -- \\
\hline & [24] & 2000 & $\mathrm{CAR}=90 \%$ \\
\hline & {$[25]$} & 1500 & car $=76.84 \%$ \\
\hline & [37] & 1760 & $\mathrm{CAR}=98.83 \%$ \\
\hline \multirow{6}{*}{ Redes neuronales } & [29] & 324 & $\mathrm{CAR}=99.07 \%$ \\
\hline & [8] & -- & -- \\
\hline & [36] & 80 & $\mathrm{CAR}=82.5 \%$ \\
\hline & [25] & 1500 & $\begin{array}{l}\text { CAR }=84.79 \% \text { para una red neuronal de propagación hacia atrás } \\
\text { CAR }=74.81 \% \text { para una red neuronal de base radial }\end{array}$ \\
\hline & [26] & 200 & CAR $=85.25 \%$ \\
\hline & [37] & 1760 & CAR $=96.46 \%$ \\
\hline \multirow{5}{*}{ Aprendizaje profundo } & [63] & 500 & $\begin{array}{l}\text { CAR }=86 \% \\
\text { F1-score }=0.8965\end{array}$ \\
\hline & [64] & 500 & $\begin{array}{l}\text { CAR }=86 \% \\
\text { F1-score }=0.8965\end{array}$ \\
\hline & {$[20]$} & 400 & $\mathrm{CAR}=92.08 \%$ \\
\hline & [27] & 9053 & $\mathrm{CAR}=75 \%$ \\
\hline & [28] & 1006 & F1-score $=0.87$ \\
\hline
\end{tabular}

Fuente: elaboración propia. 
En la Tabla 5, los artículos revisados se clasifican en 5 grupos según la tasa de precisión CAR y en 3 grupos de acuerdo al puntaje F1. La mayoría de las investigaciones alcanzan tasas de precisión por encima del $80 \%$ y un puntaje F1 mayor a 0.8 . Aunque la tasa de precisión CAR es una medida muy usada como criterio de evaluación, según lo manifestado en [64] no es una buena medida de rendimiento cuando se trata de datos no equilibrados como los que nos ocupan en esta revisión, ya que la medida favorece a la clase dominante, en este caso los registros que no presentan fallas y que no son el foco principal en la evaluación de pavimentos. La puntuación F1 se considera una medida de rendimiento más apropiada, ya que combina la tasa de detección y la tasa de falsas alarmas, dos características valiosas para evaluar la aplicabilidad de un detector del mundo real.

Tabla 5. Nivel de precisión

\begin{tabular}{|c|c|c|}
\hline \multicolumn{2}{|c|}{ Criterio de evaluación } & Autor(es) \\
\hline \multirow[t]{5}{*}{ CAR } & Menor a $80 \%$ & [27] [57] \\
\hline & $80 \%-85 \%$ & {$[36][44]$} \\
\hline & $85 \%-90 \%$ & {$[26][43][42][60][63]$} \\
\hline & $90 \%-95 \%$ & {$[11][21][20][23][24][25][35]$} \\
\hline & Mayor a 95\% & {$[9][29][30][37]$} \\
\hline \multirow[t]{3}{*}{ F1 } & Menos de 0.8 & -- \\
\hline & $0.8-0.9$ & {$[17][63][28]$} \\
\hline & Mayor de 0.9 & {$[9]$} \\
\hline
\end{tabular}

Fuente: elaboración propia.
Entre las entidades que realizan evaluación de pavimentos no existe un sistema universal de identificación de fallas superficiales. La clasificación de los tipos de fallas se realiza por contrastación con manuales desarrollados por diferentes instituciones. La metodología Vizir (del francés, Visión Inspection de Zones et Itinéraires Á Risque) y la norma de la American Society of Testing Materials, Astm6433, son dos de los más referenciados. La metodología Vizir especifica 24 tipos de deterioro, teniendo en cuenta dos tipos de degradación, y la norma AsTM6433 especifica 19 tipos. La Tabla 6 registra el tipo de falla detectada y el porcentaje de detección. Se puede observar que la mayor parte de las investigaciones se han enfocado en detección de fisuras, mientras que otras fallas apenas se mencionan; esto hace que la base de conocimiento en técnicas de detección de fisuras sea más completa y madura. De igual forma, se evidencia la necesidad de desarrollar investigaciones para detectar otros tipos de deterioro. Las metodologías Vizir y ASTM, además de clasificar y describir los tipos de daños, también especifican tres niveles de severidad y ofrecen la recomendación para el cálculo de un índice de condición del pavimento. La Tabla 7 relaciona otras características de las investigaciones presentadas en esta revisión: la primera fila indica cuáles de las investigaciones calculan indicadores de la condición de la vía, la segunda cuáles evalúan niveles de severidad por falla y la tercera fila indica si el desarrollo se ajusta a alguna norma o manual de clasificación de fallas. Como se puede observar, solo el $4 \%$ de las investigaciones calcula indicadores de la condición de la vía, el $4 \%$ calcula niveles de severidad en las fallas y el $4 \%$ se ajusta a una norma o manual, lo que hace evidente la necesidad de ampliar la investigación en este sentido.

Generalmente se estudian las fisuras porque surgen en una etapa temprana y son el origen de los distintos tipos de deterioro de un pavimento. A partir de ello se gradúa el nivel de severidad, diferenciada en tres niveles para cada daño: bajo, 
medio y alto. Estos permiten caracterizar el grado de avance en el deterioro del pavimento. Suelen estar asociados también a distintos requerimientos de conservación, variables según los casos, que van desde no hacer nada hasta la completa reposición del pavimento. Con ello se confeccionan las normas, manuales, catálogos, etc.

Tabla 6. Tipo de falla detectada

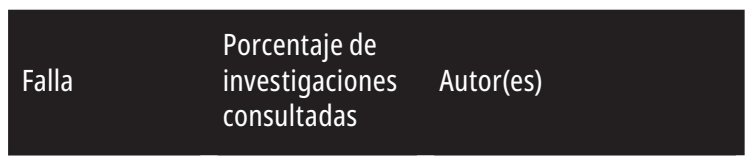

\begin{tabular}{|c|c|c|}
\hline Fisuras & $77 \%$ & $\begin{array}{l}{[5][6][7][8][10][11]} \\
{[12][15][16][17][18]} \\
{[19][20][22][23][24]} \\
{[25][27][28][29][30]} \\
{[34][35][36][37][40]} \\
{[41][44][48][58][57]} \\
{[58][59][60][63]}\end{array}$ \\
\hline Baches & $22 \%$ & $\begin{array}{l}{[12][21][26][30][37]} \\
{[40][42][45][48][62]}\end{array}$ \\
\hline Parches & $6 \%$ & [40] [43] [48] \\
\hline Ondulaciones & $4 \%$ & {$[45][46]$} \\
\hline Surcos & $4 \%$ & [12] [34] \\
\hline $\begin{array}{l}\text { Estimación de } \\
\text { la condición } \\
\text { global }\end{array}$ & $4 \%$ & [38] [39] \\
\hline Marcas viales & $4 \%$ & [12] [27] \\
\hline $\begin{array}{l}\text { Tapas de } \\
\text { alcantarilla }\end{array}$ & $2 \%$ & [9] \\
\hline
\end{tabular}

Fuente: elaboración propia.
Tabla 7. Otras características

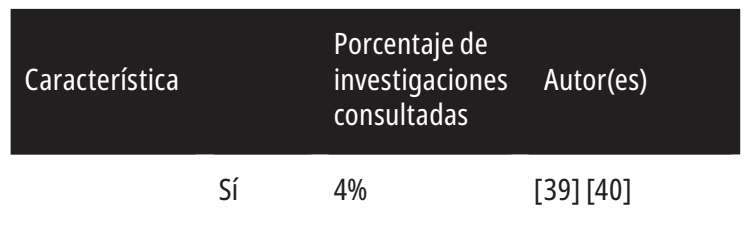

\begin{tabular}{|c|c|c|c|}
\hline $\begin{array}{l}\text { Indicadores de } \\
\text { la condición } \\
\text { de la vía }\end{array}$ & No & $96 \%$ & $\begin{array}{l}{[6][7][8][9]} \\
{[10][11][12]} \\
{[17][18][19]} \\
{[20][21][22]} \\
{[23][24][25]} \\
{[26][27][28]} \\
{[29][30][34]} \\
{[35][36][37]} \\
{[41][42][43]} \\
{[44][56][57]} \\
{[58][59][60]} \\
{[62][63][64]}\end{array}$ \\
\hline & Sí & $4 \%$ & [6] [59] \\
\hline $\begin{array}{l}\text { Niveles de } \\
\text { severidad }\end{array}$ & No & $96 \%$ & $\begin{array}{l}{[7][8][9][10]} \\
{[11][12][17]} \\
{[18][19][20]} \\
{[21][22][23]} \\
{[24][25][26]} \\
{[27][28][29]} \\
{[30][34][35]} \\
{[36][37][38]} \\
{[39][40][42]} \\
{[43][44][56]} \\
{[57][58][60]} \\
{[62][63][64]}\end{array}$ \\
\hline & Sí & $4 \%$ & [27] [64] \\
\hline $\begin{array}{l}\text { Ajustado } \\
\text { a norma o } \\
\text { manual }\end{array}$ & No & $96 \%$ & $\begin{array}{l}{[6][7][8][9]} \\
{[10][11][12]} \\
{[17][18][19]} \\
{[20][21][22]} \\
{[23][24][25]} \\
{[26][28][29]} \\
{[30][34][35]} \\
{[36][37][38]} \\
{[39][40][42]} \\
{[43][44][56]} \\
{[57][58][59]} \\
{[60][62][63]}\end{array}$ \\
\hline
\end{tabular}

Fuente: elaboración propia. 


\section{Conclusiones}

Este documento proporciona un análisis del progreso y la investigación en sistemas automáticos utilizados para la detección de fallas superficiales en pavimentos. Sobre la base de una revisión de los métodos, tecnologías y desempeño, se hacen las siguientes observaciones. (i) Para la adquisición de datos se han probado varios dispositivos (sistemas láser, cámaras de escaneo de línea, cámaras de escaneo de área, cámaras de video, sensores multiespectrales, etc.). Todos ofrecen confiabilidad bajo condiciones delimitadas, se evidencia una tendencia al uso de cámaras digitales, aunque es recomendable complementar estos datos para obtener información de profundidad y de esta manera ampliar la clasificación, incluyendo fallas que requieren esta información. (ii) Para procesar los datos y determinar la condición de la superficie, inicialmente se utilizaron técnicas de visión por computador (descomposición, semillas, umbral, detección de bordes, etc.). las investigaciones de los últimos años se han enfocado en los sistemas que utilizan aprendizaje automático (svM, árboles de decisión, redes neuronales, etc.) y las más recientes implementan específicamente el aprendizaje profundo; no obstante, las técnicas de visión por computador no fueron descartadas completamente, se han integrado en las investigaciones recientes debido a su potencial de proporcionar información valiosa que puede complementar los métodos de aprendizaje automático. (iii) La mayoría de las investigaciones alcanzan tasas de precisión por encima del $80 \%$, aunque es necesario aclarar que varias investigaciones utilizan medidas de desempeño diferentes. (iv) La detección de fallas se ha centrado en las fisuras y los baches, dejando clara la necesidad de investigación adicional en métodos que puedan detectar diferentes clases de fallas. A partir del análisis de las fisuras y la experiencia de los ingenieros viales, se detectan tempranamente las tipologías de fallas superficiales, sin necesidad de llegar a un estado de deterioro elevado y sin requerir reconstrucción total del pavimento necesitando equipos especiales.

Del análisis de los diferentes métodos, utilizados en la detección de fallas superficiales en pavimentos, se concluye que para la adquisición de los datos lo más conveniente es usar un sistema multisensorial que complemente las imágenes digitales con datos de profundidad. En cuanto al procesamiento de los datos, claramente el aprendizaje profundo es la técnica más prometedora, al ser un área en desarrollo que ofrece resultados satisfactorios.

\section{Referencias}

[1] D. E. Garzón Bejarano, Qué incidencia tiene la ausencia de infraestructura en transporte en el crecimiento económico de un país, tesis ba, Facultad de Ciencias Económicas de la Universidad Militar de Nueva Granada,Bogotá,2016.Disponibleen:https://repository. unimilitar.edu.co/bitstream/handle/10654/14198/ G a r z o n B e jar a n o D i e g o En ri que 2016 . pdf? sequence $=1 \&$ is Allowed $=y$

[2] (2017, feb. 8). "Plan Estratégico Institucional 2015 2018 V 2", Invías. [Internet]. Disponible en https:// www.invias.gov.co/index.php/archivo-y-documentos/ hechos-de-transparencia/planeacion-gestion-ycontrol/plan-estrategico-institucional/5455-planestrategico-institucional-2015-2018-v-2

[3] (2018, dic. 28). "Estado de la red vial criterio técnico segundo semestre del 2018”, Invías. [Internet]. Disponible en https:/www.invias.gov.co/index.php/ archivo-y-documentos/informacion-institucional/8397estado-de-la-red-vial-criterio-tecnico-segundosemestre-2018

[4] M. Á. Morillo Romero, Digitalización 3D con escáner de luz estructurada aplicada al área de la gestión de calidad y la conservación del patrimonio históricoartístico, tesis BA, Departamento de Física Aplicada de la Escuela Técnica Superior de Ingeniería de la Universidad de Sevilla, Sevilla, 2015.

[5] L. Bursanescu y M. Bursanescu, "Three-line high-power three-dimensional sensor", in Three-Dimensional Image Capture and Applications; ed. R. N. Ellson, vol. 3313, SPIE International Society for Optics and Photonics, 1998, pp. 105-114. doi: https://oi. org/10.1117/12.302443

[6] L. Bursanescu y M. Hamdi, "Three-dimensional laser ranging image reconstruction using three-line laser sensors and fuzzy methods", in Three-Dimensional Imaging, Optical Metrology, and Inspection V; ed. K. G. Harding, Boston: SPIE International Society for Optics and Photonics, 1999, pp. 106-117. doi: https:// doi.org/10.1117/12.370251 
[7] W. Li, J. Huyan y S. L. Tighe, "Pavement Cracking Detection Based on Three-Dimensional Data Using Improved Active Contour Model", Jour. of Transp. Eng., vol. 144, n. ${ }^{\circ}$ 2, 2018. doi: https://doi.org/10.1061/ JPEODX.0000028

[8] Y. Turkan, J. Hong, S. Laflamme y N. Puri, “Adaptive wavelet neural network for terrestrial laser scanner-based crack detection", Automat. in Const., vol. 94, pp. 191-202, 2018. doi: https://doi.org/10.1016/j. autcon.2018.06.017

[9] Y. Yu, H. Guan y Z. Ji, “Automated Detection of Urban Road Manhole Covers Using Mobile Laser Scanning Data", IEEE Transac. on Intellig. Transp. Syst., vol. 16, n. ${ }^{\circ}$, pp. 3258-3269, 2015. doi: https://doi.org/10.1109/ TITS.2015.2413812

[10] W. Ouyang y B. Xu, "Pavement cracking measurements using 3D laser-scan Images", Measur. Sci. and Tech., vol. 24, n. ${ }^{\circ}$ 10, 2013. doi: https://doi. org/10.1088/0957-0233/24/10/105204

[11] J. Huang y W. Liu, "A pavement crack detection method combining 2D with 3D information based on DempsterShafer", Comp.-Aided Civ. and Infr., vol. 29, n. ${ }^{\circ}$, pp. 299-313, 2014. doi: https://doi.org/10.1111/ mice. 12041

[12] R. Gui, X. Xu, D. Zhang, H. Lin, F. Pu, L. He y M. Cao, "A component decomposition model for 3D laser Scanning Pavement data based on high-pass filtering and sparse analysis", Sensors, vol. 18, n. ${ }^{\circ}$ 7, p. 2294, 15, jul., 2018. doi: https://doi.org/10.3390/s18072294

[13] X. M. Sun, J. P. Huang y W. Y. Liu, "Decision model in the laser scanning system for pavement crack detection", Optic. Eng., vol. 50, n. ${ }^{\circ}$ 12, pp. 107-207, 2011. doi: https://doi.org/10.1117/1.3662424

[14] H. Xing-Fei y O. Nixon, "Time Delay Integration Speeds Up Imaging”, Jour. Phot. Spect., vol. 46, n. ${ }^{\circ}$, p. 50, may. 2012.

[15] M. Yao, Z. Zhao, X. Yao y B. Xu, "Fusing complementary images for pavement cracking measurements", Measur. Sci. and Tech., vol. 26, n. ${ }^{\circ} 2$, 2015. doi: https:// doi.org/10.1088/0957-0233/26/2/025005

[16] X. Yao, M. Yao y B. Xu, "Automated Measurements of Road Cracks Using Line-Scan Imaging", Jour. of Test. and Eval., vol. 39, n. ${ }^{\circ}$ 4, pp. 621-629, 2011. doi: https://doi.org/10.1520/JTE103331

[17] L. Qingquan, Z. Qin, Z. Daqiang y M. Qingzhou, "FoSA: F* seed-growing approach for crack-line detection from pavement images", Imag. and Vis. Comp., vol. 29 , n. ${ }^{\circ} 12$, pp. 861-872, 2011. doi: https://doi.org/10.1016/j.imavis.2011.10.003
[18] Y. Huang y B. Xu, "Automatic inspection of pavement cracking distress", Jour. Of Elect. Imag., vol. 15, n. ${ }^{\circ} 1$, pp. 13-17, 2006. doi: https://doi.org/10.1117/1.2177650

[19] M. Gavilán, D. Balcones, O. Marcos, D. F. Llorca, M. A. Sotelo, I. Parra, M. Ocaña, P. Aliseda, P. Yarza y A. Amírola, "Adaptive Road Crack Detection System by Pavement Classification", Sensors, vol. 11, n. ${ }^{\circ}$ 10, pp. 9628-9657, 2011. doi: https://doi.org/10.3390/ s111009628

[20] N.-D. Hoang, Q.-L. Nguyen y V.-D. Tran, "Automatic recognition of asphalt pavement cracks using metaheuristic optimized edge detection algorithms and convolution neural network", Automat. in Const., vol. 94, pp. 203-213, 2018. doi: https://doi.org/10.1016/j.autcon.2018.07.008

[21] M. H. Yousaf, K. Azhar, F. Murtaza y F. Hussain, "Visual analysis of asphalt pavement for detection and localization of potholes", Adv. Eng. Informatics, vol. 38, pp. 527-537, 2018. doi: https://doi.org/10.1016/j. aei.2018.09.002

[22] F. Meng y A. Li, "Pavement crack detection using Sketch Token", Proced. Comp. Sci., vol. 139, pp. 151-157, 2018. doi: https://doi.org/10.1016/j. procs.2018.10.231

[23] N.-D. Hoang, "Classification of asphalt pavement cracks using laplacian pyramid-cased image processing and a hybrid computational approach", Comput. Intellig. and Neurosci., vol. 2018, 2018. doi: https://doi. org/10.1155/2018/1312787

[24] N.-D. Hoang y Q.-L. Nguyen, "Fast local laplacian-based steerable and sobel filters integrated with adaptive boosting classification tree for automatic recognition of asphalt pavement cracks", $A d v$. in Civ. Eng., vol. 2018, pp. 1-17, 2018. doi: https://doi. org/10.1155/2018/5989246

[25] N.-D. Hoang y Q.-L. Nguyen, "Automatic recognition of asphalt pavement cracks based on image processing and machine learning approaches: Comparative study on classifier performance", Math. Prob. in Eng., vol. 2018, pp. 1-16 2018. doi: https://doi. org/10.1155/2018/6290498

[26] N.-D. Hoang, "An artificial intelligence method for asphalt pavement pothole detection using least squares support vector machine and detection", $A d v$. in Civ. Eng., vol. 2018, pp. 1-12, 2018. doi: https://doi. org/10.1155/2018/7419058

[27] H. Maeda, Y. Sekimoto, T. Seto, T. Kashiyama y H. Omata, "Road damage detection using deep neural networks with images captured through a smartphone", Comp.-Aided Civ. and Infrastruc. Eng., vol. 33, 
n. ${ }^{\circ} 12$, pp. 1127-1141, 2018. doi: https://doi.org/10.1111/ mice. 12387

[28] Q. Zou, Z. Zhang, Q. Li, X. Qi, Q. Wang y S. Wang, "DeepCrack: learning hierarchical convolutional features for crack detection", IEEE Transact. on Imag. Process., vol. 28, n. ${ }^{\circ}$ 3, pp. 1498-1512, 2019. doi: https:// doi.org/10.1109/TIP.2018.2878966

[29] F. M. Nejad y H. Zakeri, "An optimum feature extraction method based on Wavelet-Radon transform and dynamic neural network for pavement distress classification", Expert Syst. with Applic., vol. 38, n. ${ }^{\circ}$ 8, pp. 9442-9460, 2011. doi: https://doi.org/10.1016/j. eswa.2011.01.089

[30] F. M. Nejad y H. Zakeri, "A comparison of multi-resolution methods for detection and isolation of pavement distress", Exp. Syst. with Applic., vol. 38, n. ${ }^{\circ}$ 3, pp. 2857-2872, 2010. doi: https://doi.org/10.1016/j. eswa.2010.08.079

[31] L. Inzerillo, G. D. Mino y R. Roberts, "Image-based $3 \mathrm{D}$ reconstruction using traditional and uav datasets for analysis of road pavement distress", Automat. in Const., vol. 96, pp. 457-469, 2018. doi: https://doi.org/10.1016/j.autcon.2018.10.010

[32] M. Stanie y P. Czech, "Self-correcting neural network in road pavement diagnostics", Automat. in Const., vol. 96, pp. 75-87, 2018. doi: https://doi.org/10.1016/j. autcon.2018.09.001

[33] R. J. Dobson, T. Colling, C. Brooks, C. Roussi, M. Kueber y D. Dean, "Collecting Decision Support System Data Through Remote Sensing of Unpaved Roads", Transp. Res. Rec.: Jour. of the Transp. Res. Board, vol. 2433, n. ${ }^{\circ}$, pp. 108-115, 2014. doi: https:// doi.org/10.3141/2433-12

[34] S. Zhang, C. D. Lippitt y S. M. Bogus, “Characterizing pavement surface distress conditions with hyper-spatial resolution natural color aerial photography", Remote Sens., vol. 8, n. ${ }^{\circ}$ 5, p. 392, 2016. doi: https://doi. org/10.3390/rs8050392

[35] H. Zakeri, F. M. Nejad y A. Fahimifar, "Rahbin: A quadcopter unmanned aerial vehicle based on a systematic image processing approach toward an automated asphalt pavement inspection", Automat. in Const., vol. 72, n. ${ }^{\circ}$ 2, pp. 211-235, 2016. doi: https://doi. org/10.1016/j.autcon.2016.09.002

[36] N. Shatnawi, "Automatic pavement cracks detection using image processing techniques and neural network", Internat. Jour. of Adv. Comp. Sci. and Applic., vol. 9, n. ${ }^{\circ}$ 9, pp. 399-402, 2018. doi: https://doi. org/10.14569/IJACSA.2018.090950
[37] Y. Pan, X. Zhang, G. Cervone y L. Yang, "Detection of asphalt pavement potholes and cracks based on the unmanned aerial vehicle multispectral imagery", IEEE Jour. of Select. Top. in Appl. Earth Observ. and Remote Sens., vol. 11, n. ${ }^{\circ}$ 10, pp. 3701-3712, 2018. doi: https://doi.org/10.1109/JSTARS.2018.2865528

[38] S. Zhang, S. M. Bogus, C. D. Lippitt, P. R. Neville, G. Zhang, C. Chen y V. Valentin, "Extracting pavement surface distress conditions based on high spatial resolution multispectral digital aerial photography", Photogram. Eng. \& Remote Sens., vol. 81, n. ${ }^{\circ}$ 9, pp. 709-720, 2015. doi: https://doi.org/10.14358/PERS.81.9.709

[39] S. Pascucci, C. Bassani, A. Palombo, M. Poscolieri y R. Cavalli, "Road asphalt pavements analyzed by airborne thermal remote sensing: preliminary results of the Venice highway", Sensors (Basel), vol. 8, n. ${ }^{\circ}$ 2, pp. 1278-1296, 2008. doi: https://doi.org/10.3390/s8021278

[40] M. Ribeiro Resende, L. L. Bariani Bernucci y J. A. Quintanilha, "Monitoring the condition of roads pavement surfaces: proposal of methodology using hyperspectral images", Jour. of Transp. Lit., vol. 8, n. ${ }^{\circ}$, pp. 201-220, 2014. doi: https://doi.org/10.1590/S223810312014000200009

[41] H. Lokeshwor, L. K. Das y S. Goel, "Robust method for automated segmentation of frames with/without distress from road surface video clips", Jour. of Transp. Eng., vol. 140, n. ${ }^{\circ}$ 1, pp. 31-41, 2014. doi: https://doi.org/10.1061/(ASCE)TE.1943-5436.0000564

[42] C. Koch y I. Brilakis, "Pothole detection in asphalt pavement images", Adv. Eng. Inform., vol. 24, n. 3 , 2011. doi: https://doi.org/10.1016/j.aei.2011.01.002

[43] G. M. Hadjidemetriou, P. A. Vela y S. E. Christodoulou, "Automated pavement patch detection and quantification using support vector", Jour. of Comp. in Civ. Eng., vol. 32, n. ${ }^{\circ}$ 1, 2018. doi: https://doi.org/10.1061/ (ASCE)CP.1943-5487.0000724

[44] W. Y. Yan y X.-X. Yuan, "A low-cost video-based pavement distress screening system for low-volume roads", Jour. of Intellig. Transp. Syst., vol. 22, n. ${ }^{\circ}$ 5, pp. 376-389, 2018. doi: https://doi.org/10.1080/154724 50.2017 .1366320

[45] R. Madli, S. Hebbar y P. Pattar, "Automatic detection and notification of potholes and humps on roads to aid drivers", IEEE Sens. Jour., vol. 15, n. ${ }^{\circ}$, pp. 4313-4318, 2015. doi: https://doi.org/10.1109/JSEN.2015.2417579

[46] M. Bellone y G. Reina, "Pavement distress detection and avoidance for intelligent vehicles", Internat. Jour. of Vehic. Autonom. Syst., vol. 13, n. ${ }^{\circ}$ 2, pp. 152-167, 2016. doi: https://doi.org/10.1504/IJVAS.2016.078810 
[47] M. Tan, S. Liang y X. Y. Li, "Design of a low-cost detecting and locating system for pavement distresses based on vibration acceleration signal", ICIC Expr. Lett., 2016.

[48] M. R. Jahanshahi, F. Jazizadeh, S. F. Masri y B. Becerik-Gerber, "Unsupervised approach for Autonomous pavement defect detection and quantification using an inexpensive depth sensor", Jour. of Comp. in Civ. Eng., vol. 27, n. ${ }^{\circ}$ 6, pp. 743-754, 2013. doi: https://doi. org/10.1061/(ASCE)CP.1943-5487.0000245

[49] (2020). "Fugro". [Internet]. Disponible en: https:// www.fugro.com/our-services/asset-integrity/roadware/aran-automatic-road-analyzer\#tabbed1. [Último acceso: 11 agosto 2018].

[50] (2015). "The digital highway data vehicle (DHDV)". [Internet]. Disponible en: http://www.pvision3d.com/ Home/DHDV .

[51] (2020). "Leica Geosystems". [Internet]. Disponible en: https://leica-geosystems.com/products/mobile-sensor-platforms/capture-platforms/leica-pegasus two-ultimate.

[52] (2019, ag. 31). Pasco. [Internet]. Disponible en: https:// www.pasco.co.jp/eng/products/real/.

[53] (2020). Dynatest. [Internet]. Disponible en: https:// www.dynatest.com/multi-functional-vehicle-mfv.

[54] (2020) Raurosgroup. [Internet]. Disponible en: http:// www.raurosgroup.com/Servicios.

[55] A. González, F. Martínez, A. Pernia, F. Alba, M. Castejon, J. Ordieres y E. Vergara, Técnicas y algortimos básicos de visión artificial, España: Servicio de Publicaciones Universidad de la Rioja, 2006.

[56] P. Subirats, J. Dumoulin, V. Legeay y D. Barba, “Automation of pavement surface crack detection using the continuous wavelet transform", Imag. Process., vol. 1, n. ${ }^{\circ}$ 1, pp. 3037-3040, 2006. doi: https://doi.org/10.1109/ ICIP.2006.313007

[57] A. Ayenu-Prah y N. Attoh-Okine, "Evaluating pavement cracks with bidimensional empirical mode decomposition", Eurasip Jour. on Adv. in Signal Process., vol. 2008, p. 7, 2008. doi: https://doi. org/10.1155/2008/861701

[58] J. Wang y R. X. Gao, "Pavement distress analysis based on dual-tree complex wavelet transform", Internat. Jour. of Pavem. and Tech., vol. 5, n. ${ }^{\circ}$ 5, pp. 283288, sept., 2012.

[59] B. Javidi, J. Stephens, S. Kishk, T. Naughton, J. McDonald y A. Isaac, "Pilot for automated detection and classification of road surface degradation features",
Reporte Técnico JHR 03-293, Connecticut Transportation Institute, University of Connecticut, 2003.

[60] L. Qingquan y L. Xianglong, "A model for segmentation and distress statistic of massive pavement images based on multi-scale strategies", Rem. Sens. and Spat. Inf. Sci., vol. 37, pp. 63-67, 2008.

[61] H. Edwards, How Machines Learn. [Libro digital], IntelligentsIA Research, 2016.

[62] J. Lin y. Liu, "Potholes detection based on svm in the pavement distress image", en Proceedings - 9th International Symposium on Distributed Computing and Applications to Business, Engineering and Science (dcabes), pp. 544-547, Hong Kong, China: IEEE ag. 2010. doi: https://doi.org/10.1109/DCABES.2010.115

[63] L. Zhang, F. Yang, Y. D. Zhang y. J. Zhu, "Road crack detection using deep convolutional neural network", en 2016 ieee International Conference on Image Processing (ICIP), Phoenix, 2016. doi: https://doi. org/10.1109/ICIP.2016.7533052

[64] M. Eisenbach, R. Stricker, D. Seichte, K. Amende, K. Debes, M. Sesselmann, D. Ebersbach, U. Stoeckert y H.-M. Gross, "How to get pavement distress detection ready for deep learning? A systematic approach", en 2017 International Joint Conference on Neural Networks (IJCNN), Anchorage, usa, 2017. doi: https://doi. org/10.1109/IJCNN.2017.7966101

[65] S. Haykin, Neural Networks and Learning Machines, New York: Prentice Hall, 2009.

[66] Guerra, A, "Aprendizaje automático: árboles de decisión", Universidad Veracruzana, Facultad de Física e Inteligencia Artificial, Maestría en Inteligencia Artificial, Notas de Clases, 2004.

[67] S. Mokhtari, Analytical Study of Computer Vision-Based Pavement Crack Quantification Using Machine Learning Techniques, tesis Ph. D., Departamento de ingeniería civil, ambiental y de construcción, College of Engineering and Computer Science, University of Central Florida, Orlando, Florida, 2015. 